Trauma Berufskrankh 2018·20 (Suppl 4):S285-S291 https://doi.org/10.1007/s10039-018-0369-8 Online publiziert: 27. April 2018

(c) Springer Medizin Verlag GmbH, ein Teil von Springer Nature 2018

CrossMark

\section{Coenen ${ }^{1} \cdot$ C. Dereskewitz ${ }^{2}$}

${ }^{1}$ Lehrstuhl für Public Health und Versorgungsforschung, Institut für Medizinische Informationsverarbeitung, Biometrie und Epidemiologie - IBE, Forschungseinheit für Biopsychosoziale Gesundheit, Ludwig-Maximilians-Universität München, München, Deutschland

${ }^{2}$ Abteilung für Handchirurgie, Plastische und Mikrochirurgie, BG Klinikum Hamburg, Hamburg, Deutschland

\title{
ICF Core Sets der Hand
}

\section{Entwicklung und Anwendung in der klinischen Praxis}

\begin{abstract}
Die Internationale Klassifikation der Funktionsfähigkeit, Behinderung und Gesundheit (ICF) bietet ein Rahmenkonzept zur Beschreibung von Funktionsfähigkeit und Kontextfaktoren. Um die Anwendung der ICF in der handchirurgischen Akut- und rehabilitativen Versorgung zu unterstützen, wurden ICF Core Sets, Kurzlisten mit ausgewählten ICF-Kategorien, entwickelt. Basierend auf dem ICF Core Set der Hand konnte das ICF Hand ${ }_{A}$, ein standardisiertes Assessment für Verletzungen und Erkrankungen der Hand, entwickelt und in der klinischen Routine über Schnittstellen der Versorgung hinweg implementiert werden.
\end{abstract}

\section{Hintergrund}

Die Wiederherstellung der Funktionsfähigkeit nach Verletzungen oder Erkrankungen der Hand ist vorrangiges Ziel akutmedizinischer und rehabilitativer Versorgung. Ein umfassendes Verständnis darüber, wie sich Verletzungen und Erkrankungen der Hand auf die Gesundheit und Funktionsfähigkeit und damit das alltägliche Leben der Betroffenen auswirken, ist wichtige Voraussetzung v. a. für die rehabilitative Versorgung. Die Weltgesundheitsorganisation (WHO) bietet mit der Internationalen Klassifikation der Funktionsfähigkeit, Behinderung und Gesundheit (ICF) [1] und deren biopsychosozialem Gesundheitsverständnis ein ideales Rahmenkonzept, um Funktionsfähigkeit und
Gesundheit von Patienten mit Verletzungen oder Erkrankungen umfassend und gleichzeitig differenziert abzubilden. Das biopsychosoziale Modell setzt sich aus den Teilen Gesundheitsstörung - kodiert über die Internationale statistische Klassifikation der Krankheiten und verwandter Gesundheitsprobleme (ICD) -, Funktionsfähigkeit - definiert über Körperfunktionen, Körperstrukturen sowie Aktivitäten und Partizipation und Kontextfaktoren, nämlich Umweltfaktoren und personbezogene Faktoren - zusammen (• Abb. 1).

Um die Anwendung der ICF mit ihren über 1400 ICF-Kategorien in der klinischen Praxis und Forschung zu unterstützen, wurden in Kooperation zwischen der WHO, dem Lehrstuhl für Public Health und Versorgungsforschung (vormals: Institut für Gesundheits- und Rehabilitationswissenschaften) am Institut für Medizinische Informationsverarbeitung, Biometrie und Epidemiologie IBE der Ludwig-Maximilians-Universität (LMU) München und der Schweizer Paraplegiker Forschung für eine Reihe von Gesundheitsstörungen sog. ICF Core Sets entwickelt [2]. ICF Core Sets sind Listen mit ICF-Kategorien, die für Personen mit einer bestimmten Gesundheitsstörung relevant sind. Die Entwicklung von ICF Core Sets folgt einer standardisierten evidenzbasierten Methodik mit 4 Vorstudien und einem mehrstufigen Konsensusprozess sowie einer sich anschließenden Validierungsphase (•Abb. 2; [3]). Seit 2002 wurden insgesamt 26 ICF Core Sets für bestimmte
Gesundheitsstörungen entwickelt. Eine Übersicht der bisher entwickelten ICF Core Sets sind der Homepage der ICF Research Branch zu entnehmen (https:// www.icf-research-branch.org/icf-coresets-projects2). Eines der ICF Core Sets ist das ICF Core Set der Hand. Mit der Entwicklung des ICF Core Sets der Hand wurde erstmals ein ICF Core Set für verschiedene Verletzungen und Erkrankungen entwickelt, die sich auf eine anatomische Struktur - die Hand beziehen.

\section{Entwicklung und Validierung des ICF Core Sets der Hand}

In den Jahren 2008 bis 2010 wurden im Rahmen eines von der Berufsgenossenschaft für Gesundheitsdienst und Wohlfahrtspflege organisatorisch betreuten Forschungsvorhabens zu Beugesehnenverletzungen die ICF Core Sets der Hand entwickelt. Dabei fungierten neben der WHO und der LMU München das BG Klinikum Hamburg, das BG Klinikum Bergmannstrost Halle, die BG Klinik Tübingen und das Unfallkrankenhaus Berlin als Kooperationspartner. ${ }^{1}$ Die Entwicklung folgte der oben dargestellten Methodik. Im Einzelnen wurden die 4 Vorstudien durchgeführt:

\footnotetext{
1 Die Deutsche Gesetzliche Unfallversicherung (DGUV) hat das Forschungsvorhaben insgesamt und auch die Erstellung und Validierung der ICF Core Sets der Hand unterstützt.
} 


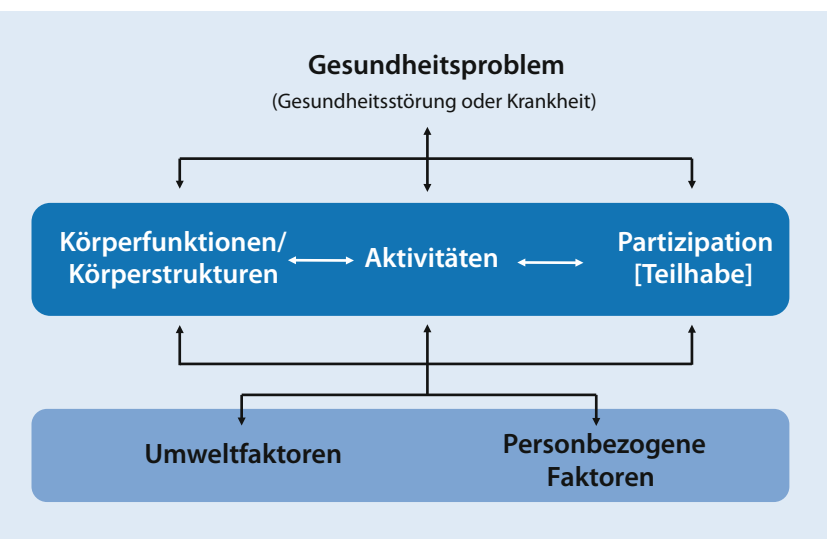

Abb. $1<$ Biopsychosoziales Modell der ICF (Internationale Klassifikation der Funktionsfähigkeit, Behinderung und Gesundheit)

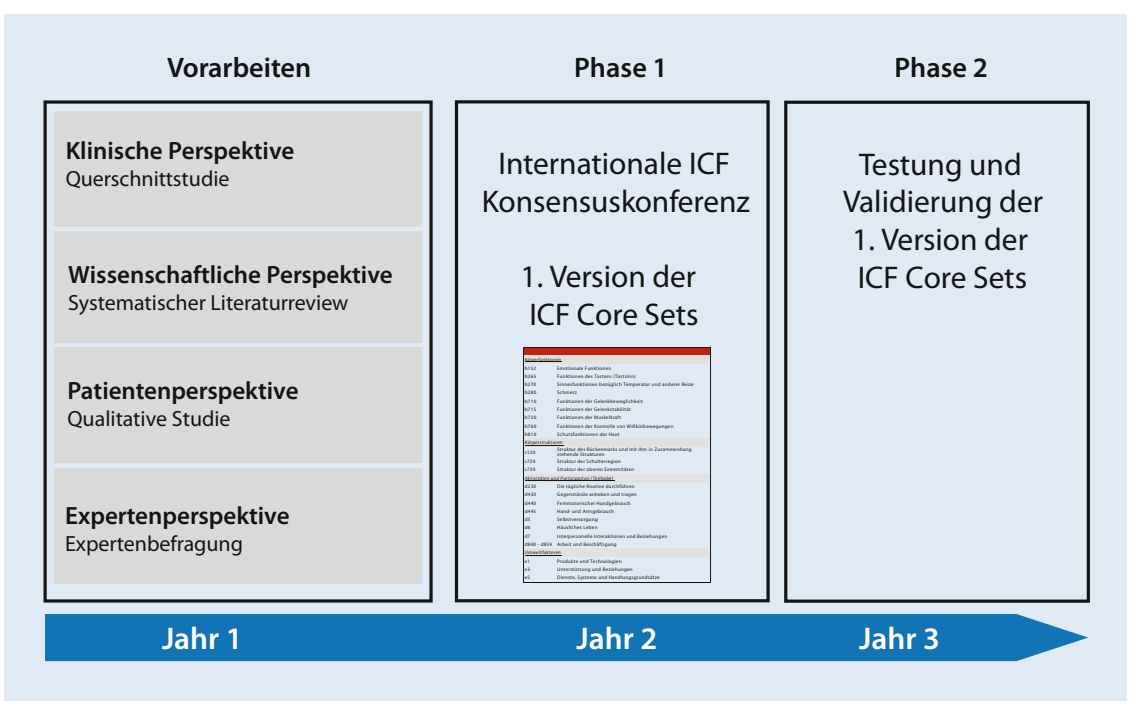

Abb. 2 \& Entwicklungsprozess von ICF (Internationale Klassifikation der Funktionsfähigkeit, Behinderung und Gesundheit) Core Sets

ICF HandA - ein mehrstufiges Verfahren

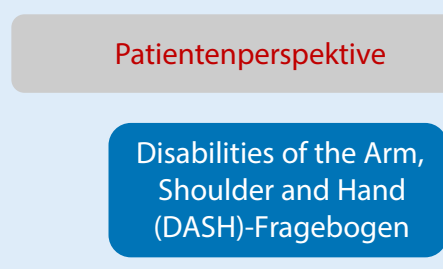

Klinische Perspektive

Screening

Spezifische Diagnostik

Abb. 3 ॥ Aufbau des ICF-basierten (ICF: Internationale Klassifikation der Funktionsfähigkeit, Behinderung und Gesundheit) Assessments Hand (ICF Hand A $_{A}$

1. eine empirische nationale Querschnittstudie mit 210 Patienten in den 4 beteiligten Kliniken,

2. ein systematischer Literaturreview der internationalen wissenschaftlichen Literatur über den Zeitraum
4. eine internationale Expertenbefragung mit 162 Experten unterschiedlicher Berufsgruppen (Ärzte, Therapeuten, Psychologen, Pflegefachkräfte) aus 59 Ländern.

Ziel dieser 4 Studien war es, aus verschiedenen Perspektiven und unter $\mathrm{Zu}$ hilfenahme von verschiedenen Methoden Aspekte der Funktionsfähigkeit und Kontextfaktoren zu identifizieren, die für Personen mit Verletzungen und Erkrankungen der Hand relevant sind [5]. Die Ergebnisse dieser Studien wurden auf einer zweitägigen internationalen Konsensuskonferenz im Mai 2009vorgestellt und dienten als Grundlage für die Entscheidungsfindung im mehrstufigen Konsensusprozess. An der Konferenz und dem Entscheidungsprozess wirkten 23 Experten aus 22 Ländern mit $[6,7]$. Als Ergebnis des Konsensusprozesses wurden verabschiedet:

1. das Kurze ICF Core Set der Hand

(23 ICF-Kategorien) als internatio-

naler Standard zur Beschreibung der

Funktionsfähigkeit von Personen mit Verletzungen und Erkrankungen der Hand im Rahmen der medizinischen Versorgung (Akutversorgung bis Rehabilitationsmaßnahmen) sowie in verschiedenen Bereichen (z. B. Leistungsträger, Gesundheitsstatistik, Lehre, klinische oder epidemiologische Forschung) (- Tab. 1) und

2. das Umfassende ICF Core Set der Hand (117 ICF-Kategorien) als internationaler Standard zur Erfassung der Funktionsfähigkeit von Personen mit Verletzungen und Erkrankungen der Hand im multidisziplinären Assessment durch verschiedene Berufsgruppen, die an der Versorgung beteiligt sind.

Beide ICF Core Sets der Hand wurden in den Jahren 2009 und 2010 in einer multizentrischen Studie an 260 Patienten validiert und liegen seit Oktober 2010 in ihrer endgültigen Version vor [8, 9]. Mit den ICF Core Sets der Hand existieren Listen mit ICF-Kategorien, die angeben, welche Aspekte der Funktionsfähigkeit und Umweltfaktoren bei Personen mit Verletzungen und Erkrankungen der Hand erfasst werden sollen. Sie legen allerdings 
nicht fest, wie diese Aspekte der Funktionsfähigkeit und Umweltfaktoren erfasst werden können und wie eine Implementierung in die klinische Versorgungsroutine $\mathrm{zu}$ erfolgen hat.

\section{Implementierung der ICF Core Sets der Hand}

Um die Implementierung der ICF Core Sets der Hand in der klinischen Praxis zu unterstützen, wurde 2011 das Nachfolgeprojekt „Implementierung der ICF Core Sets der Hand in der klinischen Praxis: Leuchtturmprojekt Hand“ initiiert. Das Projekt wurde federführend vom BG Klinikum Hamburg und dem Lehrstuhl für Public Health und Versorgungsforschung der LMU München und unter Beteiligung von 9 weiteren Kliniken (BG Universitätsklinikum Bergmannsheil Bochum, BG Klinikum Bergmannstrost Halle, Unfallkrankenhaus Berlin, BG Klinikum Duisburg, BG Unfallklinik Frankfurt am Main, BG Klinik Ludwigshafen, BG Unfallklinik Murnau, BG Klinik Tübingen und Klinik für Handchirurgie der RhönKlinikum AG Bad Neustadt a. d. Saale) durchgeführt. Im Fokus dieses Projektes stand die Anwendung des Kurzen ICF Core Sets der Hand in der täglichen Behandlung und Therapie von Patienten mit jedweden Verletzungen und Erkrankungen der Hand. Konkret angestrebt wurde, ICF-basierte Instrumente und Arbeitshilfen $\mathrm{zu}$ entwickeln und diese im (berufsgenossenschaftlichen) Heilverfahren bei Patienten mit Verletzungen oder Erkrankungen der Hand einzusetzen. Die Transparenz des Rehabilitationsverfahrens für alle am Prozess Beteiligten sollte dabei durch die gemeinsame Sprache der ICF erhöht und Abläufe sollten nachvollziehbar gestaltet werden.

Um das Kurze ICF Core Set der Hand als Steuerungsinstrument in den handchirurgischen Abteilungen der Kliniken zu implementieren, wurden im Rahmen des Projektes die nachfolgend genannten Arbeiten und Aufgaben durchgeführt.
Trauma Berufskrankh 2018 20 (Suppl 4):S285-S291

https://doi.org/10.1007/s10039-018-0369-8

(c) Springer Medizin Verlag GmbH, ein Teil von Springer Nature 2018

\section{Coenen · C. Dereskewitz}

\section{ICF Core Sets der Hand. Entwicklung und Anwendung in der klinischen Praxis}

\section{Zusammenfassung}

Die Internationale Klassifikation der Funktionsfähigkeit, Behinderung und Gesundheit (ICF) und ihr biopsychosoziales Gesundheitsverständnis bieten ein ideales Rahmenkonzept, um Funktionsfähigkeit und Gesundheit von Personen mit Gesundheitsstörungen umfassend abzubilden und zu beschreiben. Um die Anwendung der ICF in der klinischen Routine zu unterstützen, wurden für Verletzungen und Erkrankungen der Hand ICF Core Sets der Hand, Kurzlisten mit relevanten ICF-Kategorien, evidenzbasiert entwickelt und anschließend validiert. Im "Leuchtturmprojekt Hand" konnte, basierend auf den ICF Core Sets der Hand, ein ICF-basiertes Assessment (ICF Hand $d_{A}$ ) entwickelt werden. Dieses wurde mit Behandlungsstandards und einem standardisierten Berichtswesen in einer prospektiven Längsschnittstudie erprobt. Basierend auf Daten aus dem ICF Hand , $_{\text {, }}$ konnte ein zusammenfassender Score der Funktionsfähigkeit (ICF Score Hand) entwickelt werden. Mit dem ICF Hand sowie den anderen im Projekt entwickelten Materialien kann die Dokumentation und Erfolgskontrolle der akutmedizinischen und rehabilitativen Versorgung von Patienten mit Verletzungen und Erkrankungen der Hand um Aspekte der Funktionsfähigkeit ergänzt werden und unterstützt die ganzheitliche Sicht auf die Probleme der Patienten über die Schnittstellen der Versorgung hinweg.

Schlüsselwörter

Funktionsfähigkeit · Handverletzungen . Assessment · Akutmedizin · Rehabilitation

\section{ICF Core Sets for Hand Conditions. Development and implementation in clinical routine}

\section{Abstract}

The International Classification of Functioning, Disability and Health (ICF) and its biopsychosocial perspective provide an ideal framework to describe functioning and health of persons with health conditions. To facilitate the use of the ICF in clinical routine the ICF Core Set for Hand Conditions, short lists of relevant ICF categories for any kind of injuries and diseases located on the hand, were developed and validated. Based on the Brief ICF Core Set for Hand Conditions an ICF-based assessment (ICF Hand ${ }_{A}$ ) was established within the Lighthouse Project Hand. The ICF Hand $_{A}$ along with treatment guidelines and a standardized reporting system was used in

\section{Festlegung eines ICF-basierten Assessments}

Auf einer nationalen Konsensuskonferenz im Dezember 2012 wurden unter Beteiligung der im Projekt etablierten Arbeitskreise BG-Kliniken und DGUV (Deutsche Gesetzliche Unfallversicherung) evidenzbasiert verschiedene Verfahren (Testverfahren, Fragebögen, klinische Untersuchungen) festgelegt, um die Funktionsfähigkeit von Patienten a prospective longitudinal study. A summary score for functioning (ICF Score Hand) was developed using data assessed with the ICF Hand . The use of the ICF Hand $_{A}$ and other materials and tools developed within the Lighthouse Project Hand facilitate the documentation, reporting and monitoring of the acute and rehabilitative treatment of persons with hand injuries and diseases and support the holistic perspective on patients' problems in functioning.

\section{Keywords}

Functioning - Hand injuries - Assessment . Acute medicine $\cdot$ Rehabilitation mit Verletzungen und Erkrankungen der Hand standardisiert zu erfassen. Mit diesem ICF-basierten Assessment - ICF Hand $_{\mathrm{A}}$ - können alle Aspekte der Funktionsfähigkeit über ein Screening und bei Bedarf mit spezifischen Testverfahren erfasst und dokumentiert werden (• Abb. 3; [10]). Im Behandlungsverlauf können so auch über Patientengruppen hinweg Daten verglichen und Verläufe abgebildet werden. Alle im ICF Hand $_{\mathrm{A}}$ enthaltenen Assessments sind mit Anleitungen zur 
Tab. 1 Kurzes ICF (Internationale Klassifikation der Funktionsfähigkeit, Behinderung und Gesundheit) Core Set der Hand

\begin{tabular}{|c|c|}
\hline ICF Code & Titel \\
\hline \multicolumn{2}{|c|}{ Körperfunktionen } \\
\hline b152 & Emotionale Funktionen \\
\hline b265 & Funktionen des Tastens (Tastsinn) \\
\hline b270 & Sinnesfunktionen bezüglich Temperatur und anderer Reize \\
\hline b280 & Schmerz \\
\hline b710 & Funktionen der Gelenkbeweglichkeit \\
\hline b715 & Funktionen der Gelenkstabilität \\
\hline b730 & Funktionen der Muskelkraft \\
\hline b760 & Funktionen der Kontrolle von Willkürbewegungen \\
\hline b810 & Schutzfunktionen der Haut \\
\hline \multicolumn{2}{|c|}{ Körperstrukturen } \\
\hline s120 & Struktur des Rückenmarks und mit inm in Zusammenhang stehende Strukturen \\
\hline s720 & Struktur der Schulterregion \\
\hline s730 & Struktur der oberen Extremitäten \\
\hline \multicolumn{2}{|c|}{ Aktivitäten und Partizipation (Teilhabe) } \\
\hline $\mathrm{d} 230$ & Die tägliche Routine durchführen \\
\hline $\mathrm{d} 430$ & Gegenstände anheben und tragen \\
\hline$d 440$ & Feinmotorischer Handgebrauch \\
\hline d445 & Hand- und Armgebrauch \\
\hline d5 & Selbstversorgung \\
\hline d6 & Häusliches Leben \\
\hline d7 & Interpersonelle Interaktionen und Beziehungen \\
\hline d840-d859 & Arbeit und Beschäftigung \\
\hline \multicolumn{2}{|c|}{ Umweltfaktoren } \\
\hline e1 & Produkte und Technologien \\
\hline e3 & Unterstützung und Beziehungen \\
\hline e5 & Dienste, Systeme und Handlungsgrundsätze \\
\hline
\end{tabular}

Erfassung und Dokumentation sowie teilweise mit Foto- und Videomaterial auf der Homepage des Projektes dargestellt (http://leuchtturmprojekt-hand. de/messung-der-funktionsfaehigkeit/).

\section{Erprobung des ICF-basierten Assessments}

Im Rahmen einer multizentrischen Querschnittstudie wurde 2013 das ICF Hand $_{A}$ an 294 Patienten erprobt. Für diese Patienten konnte erstmals ein Profil der Funktionsfähigkeit, basierend auf der standardisierten Erfassung der Funktionsfähigkeit bei Patienten mit Verletzungen und Erkrankungen der Hand, erstellt werden (• Abb. 4).

\section{Entwicklung von ICF-basierten Behandlungsstandards}

Für 6 ausgewählte Erkrankungen und Verletzungen (Fingerfrakturen [dreigliedrig], Beugesehnenverletzungen, Amputationen mit Stumpfversorgung, komplexes regionales Schmerzsyndrom [CRPS], Rhizarthrose und Morbus Dupuytren) wurden im Arbeitskreis BGKliniken ICF-basierte Behandlungsstandards festgelegt. Die Behandlungsstandards legen ein weitestgehend standardisiertes Vorgehen bezüglich diagnostischem Vorgehen, Operationstechniken bzw. konservativer Behandlung und Nachbehandlung fest. Zudem werden Zeitpunkte definiert, zu denen der Einsatz des ICF Hand $_{\mathrm{A}}$ erfolgen soll. Als Vorbereitung für eine standardisierte Berichterstattung (z.B. im Rahmen der Arztbriefschreibung) wurde für alle Be- reiche des ICF Hand $_{\mathrm{A}}$ festgelegt, wie die Darstellung der Funktionsfähigkeit bei Erstbefundung und im Behandlungsverlauf $\mathrm{zu}$ erfolgen hat. Hierfür wurden Textbausteine und ein Ampelsystem zur Darstellung von Bereichen der Funktionsfähigkeit, für die kein (grün), potenzieller (orange) oder dringender (rot) Behandlungsbedarf besteht, entwickelt. Für die Darstellung von Veränderungen im Behandlungsverlauf wurden zudem für alle Aspekte der Funktionsfähigkeit Kriterien für Verbesserungen bzw. Verschlechterungen der verschiedenen Aspekte (z.B. Muskelkraft, Handgebrauch) definiert, die sich ebenfalls direkt aus den erhobenen

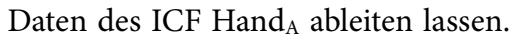

Im Rahmen der Entwicklung der Behandlungsstandards wurden auch eine detaillierte Definition und eine Systematik für Körperstrukturen (z. B. Knochen, Nerven, Arterien, Gelenke und Weichteile) ausgearbeitet, die deutlich spezifischer als die Systematik der ICF-Komponente Körperstrukturen ist. Zudem wurden grafische Darstellungen („Handskizzen“) für diese Strukturen erstellt (• Abb. 5). Mithilfe dieser Handskizzen können Schädigungen im Bereich von Körperstrukturen anhand der festgelegten Systematik standardisiert und detailliert dokumentiert werden.

\section{Entwicklung eines elektronischen Datenerfassungstools}

Für die Implementierung der ICF-basierten Behandlungsstandards in der klinischen Praxis wurde im Klinikinformationssystem medico ein elektronisches Datenerfassungstool (e-tool) entwickelt, das die Dokumentation und Steuerung der Erfassung von Daten des ICF Hand gemäß der in den Behandlungsstandards festgelegten Referenzpunkte sowie weiterer, klinisch relevanter Daten ermöglicht. Nach erfolgter Erstbefundung wird dem Kliniker direkt auf der Eingangsseite des e-tools über das Ampelsystem angezeigt, welche Bereiche der Funktionsfähigkeit bei einem Patienten problematisch sind und für welche ggf. Behandlungsbedarf besteht. Veränderungen im Behandlungsverlauf können ebenfalls über das e-tool abgebildet werden. Zudem kann 


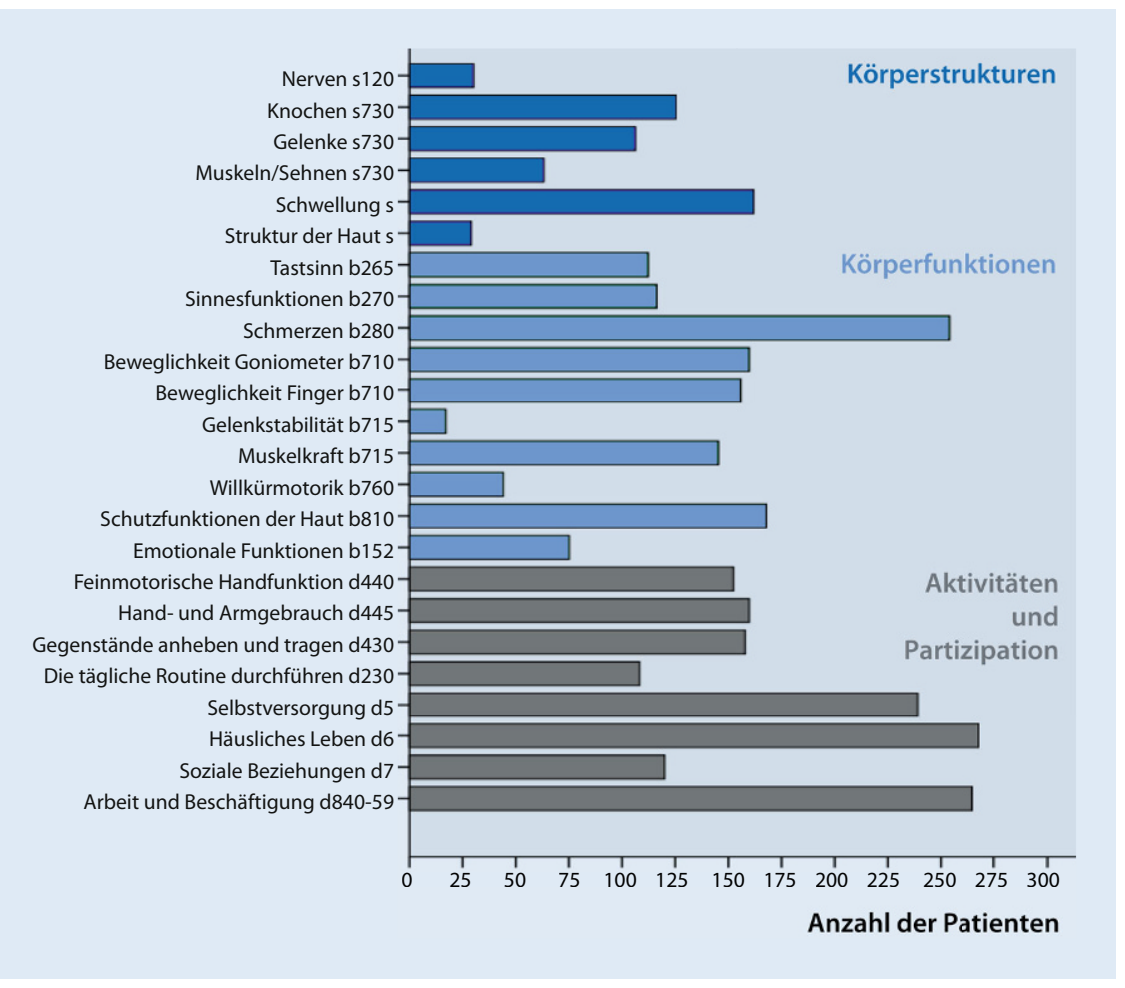

Abb. 4 ム Profil der Funktionsfähigkeit basierend auf dem ICF (Internationale Klassifikation der Funktionsfähigkeit, Behinderung und Gesundheit) $\mathrm{Hand}_{A}$

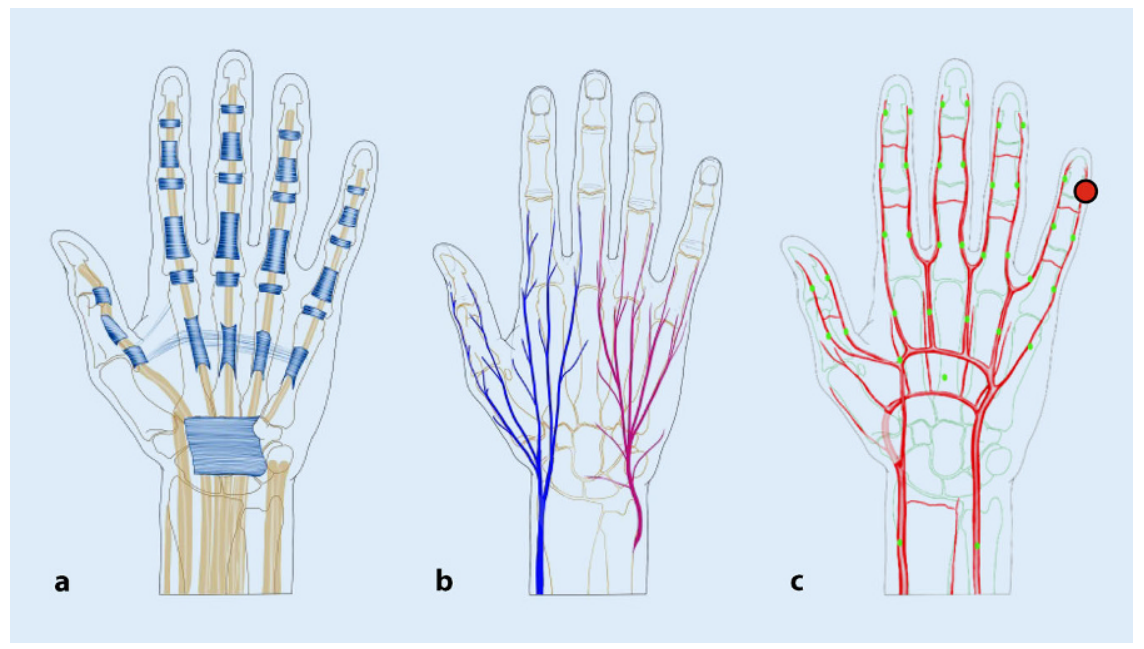

Abb. 5 ム Handskizzen für ausgewählte Strukturen: „Bänder“, „Nerven“, „Arterien“. Beispiel Befund: Komplettdurchtrennung Arterie Finger V, Höhe Endglied rechts, ulnar. a Bänder, b Nerven, c Gefäße

ein standardisierter Bericht erzeugt werden, der die wichtigsten Daten zur Funktionsfähigkeit über Textbausteine und das Ampelsystem zusammenfasst.

\section{Implementierung des \\ Behandlungsstandards}

Die Implementierung der Behandlungsstandards in der klinischen Praxis er-
Datensätze von weiteren 301 Patienten bis zu 24 Wochen nach Aufnahme in die Klinik, basierend auf dem ICF Hand $_{\mathrm{A}}$, erfasst werden.

\section{Entwicklung des ICF Score Hand}

Auf Grundlage der in den verschiedenen Studien erhobenen Daten wurde der ICF-basierte Score zur Funktionsfähigkeit der Hand (ICF Score Hand) als zusammenfassender Score zur Funktionsfähigkeit der Hand entwickelt. Mit dem ICF Score Hand steht eine Metrik von 0 (keine Beeinträchtigung der Funktionsfähigkeit) bis 100 (vollständige Beeinträchtigung der Funktionsfähigkeit) zur Verfügung, die den aktuellen Grad der (Beeinträchtigung der) Funktionsfähigkeit eines Patienten angibt. Der Score kann für jeden Patienten mit vorliegen-

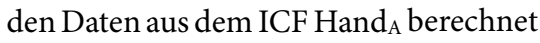
werden und so auch im Behandlungsverlauf Verbesserungen oder Verschlechterungen der Funktionsfähigkeit abbilden. Auch Vergleiche zwischen Patienten oder Patientengruppen (z. B. mit bestimmten Diagnosen) sind so möglich (•Abb.6).

\section{Bereitstellung einer Lernplattform}

Die im Verlauf des Forschungsvorhabens entwickelten Materialien werden über die Homepage des Projektes (www.leuchtturmprojekt-hand.de) zur Verfügung gestellt. Diese Materialien einschließlich eines ICF-Lernprogramms (http://leuchtturmprojekthand.de/e-learning/) können als Beispiel für die Implementierung der ICF in anderen Bereichen verwendet werden. Fünf Fallstudien illustrieren den Einsatz des ICF Hand $\mathrm{A}_{\mathrm{A}}$ und der Behandlungsstandards in der klinischen Routine (http://leuchtturmprojekt-hand.de/ fallstudien/).

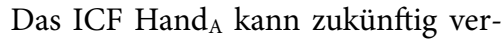
wendet werden, um die Erfassung der Funktionsfähigkeit in der ICD-11 zu unterstützen. Auch die in den verletzungsbzw. krankheitsspezifischen Behandlungsstandards festgelegten Interventionen, die sich auf konkrete Aspekte der Funktionsfähigkeit beziehen, legen bereits jetzt eine gemeinsame Verwendung der 3 WHO-Klassifikationen ICD, ICF 


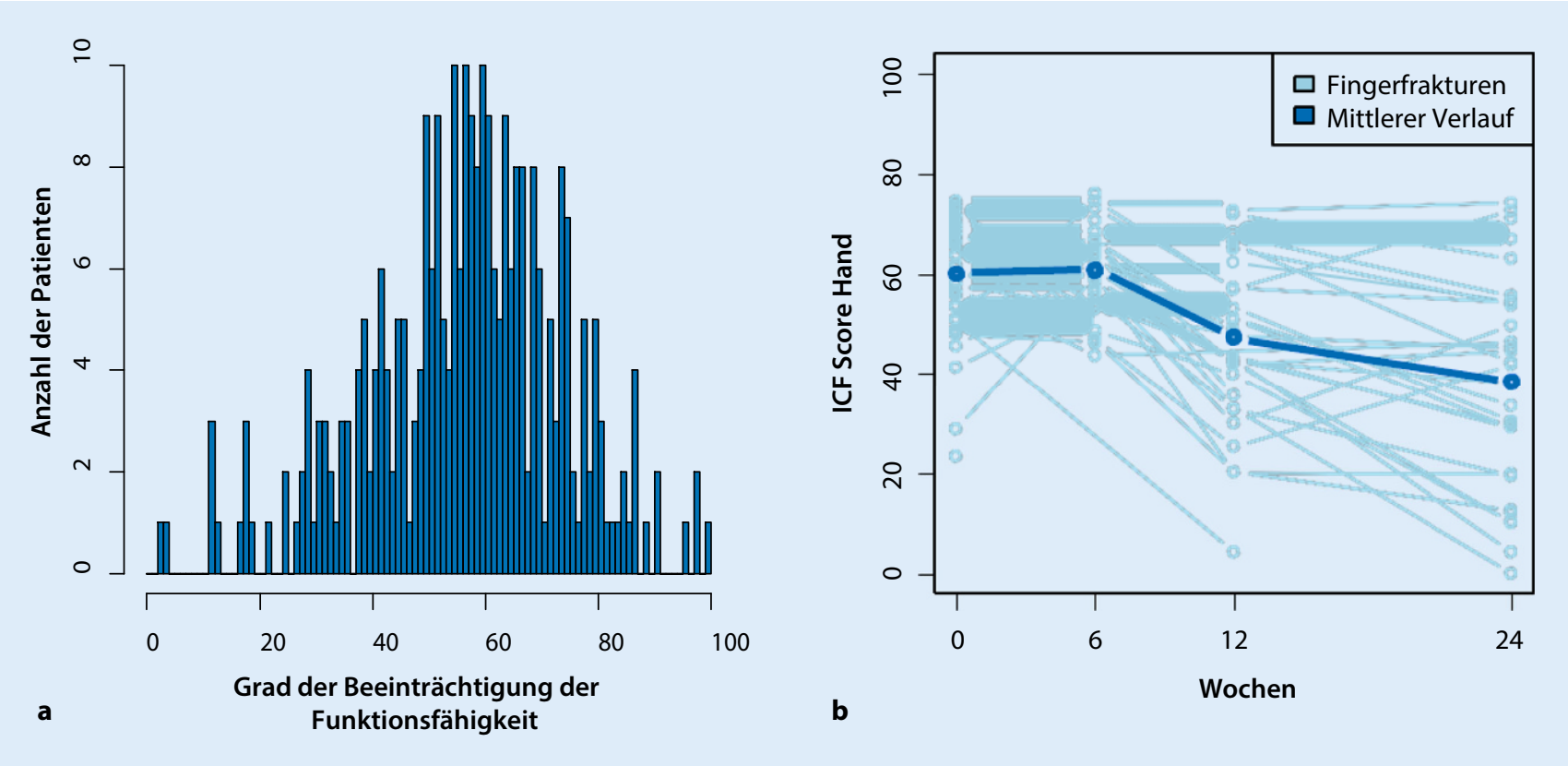

Abb. 6 ム ICF (Internationale Klassifikation der Funktionsfähigkeit, Behinderung und Gesundheit) Score Hand. a ICF Score Hand zum Zeitpunkt der Erstbefundung für verschiedene Diagnosen; Daten der prospektiven Längsschnittstudie $(N=294)$. b Entwicklung des ICF Score Hand im Verlauf (bis zu 24 Wochen) nach Erstbefunden für Patienten mit Fingerfrakturen. Daten der prospektiven Längsschnittstudie $(N=75)$

und der International Classification of Health Interventions (ICHI) nahe. Die WHO empfiehlt, diese WHO-Klassifikationen gemeinsam zu verwenden [11, 12]. In der Beta-Version der 11. Revision der ICD (ICD-11) ist dies bereits umgesetzt; hier werden in der sog. „Supplementary section for functioning " ausgewählte Aspekte von Körperfunktionen und Aktivitäten sowie Partizipation für die zukünftigen Anwender zur Verfügung gestellt [13]. Die Fallstudien des Leuchtturmprojekts Hand können als Beispiele der gemeinsamen Verwendung der WHO-Klassifikationen genutzt werden [14].

\section{Fazit für die Praxis}

- Das Leuchtturmprojekt Hand ist ein Beispiel für die Implementierung der ICF in der Patientenversorgung.

- Mit den erstellten Materialien und Tools konnte ein Erfassungs- und Steuerungsinstrument in der Akutversorgung und rehabilitativen Behandlung von Patienten mit Verletzungen und Erkrankungen der Hand implementiert werden.
- Durch die Verwendung der gemeinsamen Sprache der ICF und der Anwendung ICF-basierter Tools kann die Transparenz des Rehabilitationsverfahrens für alle am Prozess beteiligten Personen erhöht und Abläufe können nachvollziehbar gestaltet werden.

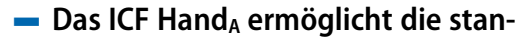
dardisierte Erfassung der Funktionsfähigkeit bei Patienten mit Verletzungen und Erkrankungen der Hand unabhängig von deren Ätiologie über den gesamten Heilverlauf, von der Akutversorgung über die rehabilitative Behandlung bis zur Wiedererlangung der Arbeitsfähigkeit. Es ermöglicht so eine ganzheitliche Betrachtung der Patienten unter Einbezug teilhabe- und aktivitätsorientierter Aspekte. 
Alle im Artikel erwähnten Studien wurden mit Zustimmung der zuständigen Ethikkommissionen, im Einklang mit nationalem Recht sowie gemäß de Deklaration von Helsinki von 1975 (in der aktuellen, überarbeiteten Fassung) durchgeführt. Von allen beteiligten Patienten liegt eine Einverständniserklärung vor.

The supplement containing this article is not sponsored by industry.

\section{Literatur}

1. World Health Organization (2001) International classification of functioning, disability and health (ICF). World Health Organization, Geneva

2. Cieza A, EwertT, Ustün TB etal (2004) Development of ICFCoreSetsfor patients with chronic conditions. JRehabil Med Suppl 44:9-11

3. Selb M, Escorpizo R, Kostanjsek N et al (2015) A guide on how to develop an international classification of functioning, disability and health core set. Eur JPhys Rehabil Med 51:105-117

4. Kus S, Van De Ven-Stevens LA, Coenen M et al (2011) What is our knowledge of functioning and disability in hand conditions based on? Arch Phys Med Rehabil 92:1326-1332

5. Kus S, Coenen M, Cieza A (2010) Die Entwicklung der ICF Core Sets der Hand - Überblick über die Vorbereitungsphase und die internationale ICF Konsensuskonferenz. Z Handther 3(1):6-15

6. Rudolf K-D, Kus S, Chung KC et al (2012) Development of the international classification of functioning, disability and health core sets for hand conditions - results of the World Health Organization International Consensus Process. Disabil Rehabil 34:681-693

7. Rudolf K-D, Kus S, Coenen M et al (2010) Report on the international ICF consensus conference on the ICF core sets for hand conditions. J Hand Ther 15:73-76

8. Kus S, Dereskewitz C, Wickert M et al (2011) Validation of the comprehensive international classification of functioning, disability and health (ICF) core set for hand conditions. J Hand Ther 16:58-66

9. Kus S, OberhauserC,Cieza A (2012)Validation of the brief International Classification of Functioning, Disability, and Health (ICF) core set for hand conditions. JHand Ther 25:274-286

10. Kus S, Dereskewitz C, Coenen M et al (2017) International Classification of Functioning, Disability and Health: development of an assessment set to evaluate functioning based on the Brief ICF Core Set for Hand Conditions - ICF HandA. J Hand Surg EurVol 42:731-741

11. Kohler F, Selb M, Escorpizo R et al (2012) Towards the joint use of ICD and ICF: a call for contribution. JRehabil Med 44:805-810

12. Selb M, Kohler F, Robinson Nicol MM et al (2015) ICD-11: a comprehensive picture of health, an update on the ICD-ICF joint use initiative. J Rehabil Med 47:2-8

13. World Health Organization (2018) ICD-11 beta draft. https://icd.who.int/browse11/I-m/en;. Zugegriffen:3. Apr. 2018

14. Coenen M, Kus S, Eisele A et al (2018) Die gemeinsame Verwendung von WHO-Klassifikationen am Beispiel von Fallstudien zu Verletzungen und Erkrankungen der Hand. 27. Rehawissenschaftliches Kolloquium. Deutsche Rentenversicherung Bund München, S70-72 